\title{
Risk factors for common mental disorders
}

\section{in women}

\author{
Population-based longitudinal study
}

VIKR AM PATEL, BETTY R. KIRKWOOD, SULOCHANA PEDNEKAR, HELEN WEISS and DAVID MABEY

\section{Background The determinants of common mental disorders in women have not been described in longitudinal studies from a low-income country.}

\section{Method Population-based cohort} study of 2494 women aged 18 to 50 years, in India. The Revised Clinical Interview Schedule was used for the detection of common mental disorders.

Results There were 39 incident cases of common mental disorder in 2166 participants eligible for analysis (12-month rate $1.8 \%, 95 \%$ Cl I.3-2.4\%). The following baseline factors were independently associated with the risk for common mental disorder: poverty (low income and having difficulty making ends meet); being married as compared with being single; use of tobacco; experiencing abnormal vaginal discharge; reporting a chronic physical illness; and having higher psychological symptom scores at baseline.

\section{Conclusions Programmes to reduce} the burden of common mental disorder in women should target poorer women, women with chronic physical illness and who have gynaecological symptoms, and women who use tobacco.

Declaration of interest None.
Common mental disorders are depressive and anxiety disorders which are typically encountered in community and primary care settings (Goldberg \& Huxley, 1992). They are the leading mental health cause of disability in the global burden of disease (World Health Organization, 2001). Crosssectional population-based studies consistently show that the poor and marginalised are at greater risk of having common mental disorders (Patel \& Kleinman, 2003). Women are at greater risk, including in low- and middle-income countries; a review of the possible explanations for this found no evidence to support a hormonal or biological mechanism (Piccinelli \& Wilkinson, 2000). Gender disadvantage and exposure to intimate-partner violence are key risk factors for common mental disorder in women (Patel et al, 2006). Surveys have also shown an association between reproductive and sexual complaints (such as the complaint of vaginal discharge) and common mental disorder (Prasad et al, 2003; Patel et al, 2006). Other risk factors, reported principally from high-income countries, include chronic physical health problems (Evans et al, 2005), tobacco misuse and alcohol misuse (Breslau et al, 2005). In this paper, we describe the results of a longitudinal population-based study of women's health in Goa, India. We hypothesised that poverty, gender disadvantage, poor physical and gynaecological health and substance misuse were risk factors for the development of new episodes of common mental disorder.

\section{METHOD}

This was a population-based cohort study in the state of Goa on India's west coast, with a population of 1.4 million. The 1998-9 National Family Health Survey reported that Goa, together with some states such as Kerala, had some of the best health indicators in the country (International Institute for Population Sciences, 2001).

\section{Sample}

The study population comprised women aged 18-45 years living in nine villages of the catchment area of the Aldona Primary Health Centre $(n=8595) ; 3000$ women were randomly selected from the population registers maintained by the health department. The eligibility criteria for recruitment were age between 18 and 50 years (since the enumeration registers were up to 4 years old in some villages); residence in the area for the next 12 months; speaking one of the study languages (Konkani, English, Hindi, Marathi); and not being currently pregnant. If a randomly selected woman did not meet all these criteria, then the researcher was instructed to replace her using a priori criteria for identifying an eligible woman (Patel et al, 2006).

\section{Recruitment and follow-up}

Recruitment took place over a 19-month period from November 2001 to May 2003. Details of the recruitment procedure and data collection have been described in earlier publications (Patel et al, 2006). In brief, the mandatory requirements for participation were a face-to-face interview with a trained researcher, and the collection of vaginal or urine specimens for the diagnosis of reproductive tract infection. Participants who consented to a gynaecological examination also had their blood pressure, weight and height measured. All recruits who consented to participate and completed the recruitment procedures were reviewed at 6 and 12 months after recruitment. Thus, there were three rounds of data collection: at recruitment, and at 6 and 12 months (reviews).

\section{Risk factors}

Risk factors were assessed at recruitment. We conducted a semi-structured interview, which was a composite of items derived from existing interviews used in other studies of reproductive and mental health in Goa. The interview was evaluated in a pilot study; interrater reliability of key variables was moderate to high (kappas from 0.58 to 0.87$)$. The data collected from these sources were organised in the following manner for the analyses of risk factors.

\section{Socio-economic risk factors}

Information on the participant's age, education, religion and marital status was collected. Economic status was measured by 
means of questions on type of housing, access to water and a toilet, household income, employment status, indebtedness and the experience of hunger due to lack of money to buy food in the previous 3 months.

\section{Psychological factors}

Two measures were used for psychological health. The Scale for Somatic Symptoms measures symptoms that are features of somatoform disorders. The scale elicits the experience of 20 common somatic symptoms in the previous 2 weeks (Chaturvedi \& Sarmukaddam, 1987), in four categories: pain-related symptoms such as headache and body ache; sensory symptoms such as hot or cold sensations and tingling; nonspecific symptoms such as tiredness and weakness; and symptoms of biological dysfunction such as poor sleep and constipation. Each symptom is rated on a Likert scale (0-2) of increasing severity. The scores of these four scales were summed to generate a somatoform disorder symptom score for each participant. The second measure was the Revised Clinical Interview Schedule (CIS-R), a structured interview for the measurement and diagnosis of common mental disorder in community and primary care settings (Lewis et al, 1992). The CIS-R is the instrument for the UK national surveys of psychiatric morbidity, and has been widely used in developing countries, including India. The Konkani language version of the CIS-R administered in the present study had been previously followed in Goa (Patel et al, 1998). The interview consists of 14 sections, each covering specific symptoms such as anxiety, depression, irritability, fatigue, obsessions, compulsions and panic. The sum of the section scores generates a total score, a measure of non-psychotic psychiatric morbidity. Scores $>11$ signify caselevel morbidity. In addition, interview data can be processed using the PROQSY software to generate ICD-10 diagnostic categories (World Health Organization, 1992).

\section{Reproductive health factors}

Participants were asked about pregnancies; numbers of pregnancies and their outcome were recorded, with more detailed history of pregnancy in the previous 12 months. Participants who were sexually active were asked about their experience of difficulty in conception in the previous 12 months. A menstrual history elicited the experience of irregular menstrual cycles and dysmenorrhoea in the previous 12 months. Participants were asked about their experience of five gynaecological symptoms (abnormal vaginal discharge, dysuria, lower abdominal pain, genital itching and dyspareunia) in the previous 3 months. Definitions of these symptom categories were derived from guidelines for reproductive health research (Jejeebhoy et al, 2003).

\section{Gender disadvantage factors}

Questions covered five domains. The first domain was elicited as part of the socioeconomic factors, i.e. marital history; being widowed or divorced poses specific disadvantages for women in South Asia. In addition, being married or having had a pregnancy during adolescence $(<20$ years old) indicate restricted productive choices. The second domain covered the lifetime experience of verbal, physical and sexual violence by the spouse and concerns about the spouse's substance use habits. Violence experienced from any other person was elicited from all participants. The third domain covered the autonomy the woman had to make decisions regarding visiting her mother's or friend's home, seeing a doctor, keeping money aside for personal use, and having time to do things for herself; the responses to these four items (each scored 0-2) were combined to generate an autonomy score (range $0-8$ ). The fourth domain enquired about the level of engagement, in the past 3 months, with four activities: religious activities, participation in a community or voluntary group, social outings to meet friends or relatives, and having friends or relatives visit her. The responses to these four items (each scored $0-4)$ were combined to generate a social integration score (range 0-16). The fifth domain consisted of items regarding support from family when faced with five different situations (good news, a personal problem, needing to borrow a small amount of money, feeling low and when ill). The responses to these five items (each scored $0-1$ ) were combined to generate a family support score (range $0-5$ ).

\section{Physical health factors}

Estimation of haemoglobin was based on a finger-prick sample of blood, using the Hemocue system (Krenzischeck \& Tanseco, 1996). Anaemia was evaluated as a categorical variable (absent, $\mathrm{Hb} \geqslant 11 \mathrm{~g} / \mathrm{dl}$; mild to moderate, $\mathrm{Hb} 8-10.9$ g/dl; severe, $\mathrm{Hb}<8 \mathrm{~g} /$ dl). The medical examination data collected from participants who consented to see the gynaecologist included weight, height and blood pressure. Body mass index (BMI) values were categorised ( $<17$; 17-19; 20-24; and $>25 \mathrm{~kg} / \mathrm{m}^{2}$ ). Items of the WHO Disability Assessment Schedule (DAS; Chwastiak \& Von Korff, 2003) that measure physical disabilities (standing for long periods, household responsibilities, walking a long distance, getting dressed, washing whole body, day-to-day work) were added to generate a physical disability score (range 6-17). The diagnosis of reproductive tract infection was established using gold-standard laboratory tests: polymerase chain reaction assay for chlamydial and gonococcal infections, culture for trichomonas vaginalis and Gram-stained slides for candidiasis and bacterial vaginosis (Meehan et al, 2003). The presence of other chronic health problems was based on selfreport of the presence of a long-standing illness; participants were asked about the nature of the illness.

\section{Outcome measures}

The CIS-R data were processed using the PROQSY software to generate ICD-10 diagnoses. The primary outcome was the presence of an ICD-10 diagnosis equivalent to a common mental disorder, i.e. any anxiety or depressive disorder, at either of the reviews.

\section{Analysis}

Analyses of the risk factors of common mental disorders were carried out for participants who completed at least one review and who did not have a common mental disorder at recruitment. Logistic regression was used for all analyses, with incident case of common disorder as the outcome. First, univariate analyses were performed for each socio-economic risk factor; all those with $P \leqslant 0.1$ were included in a multivariate model. The factors with $P \leqslant 0.1$ in this multivariate model were retained for subsequent analyses. Next, the associations of risk factors in the other domains (gender disadvantage, mental health and reproductive and physical health) with common mental disorder were estimated. The factors with $P<0.1$ in univariate analyses were adjusted for the socio-economic factors identified above; those for which $P$ remained below 0.1 , together with the socio-economic factors, formed the final multivariate model. Analysis of obstetric 


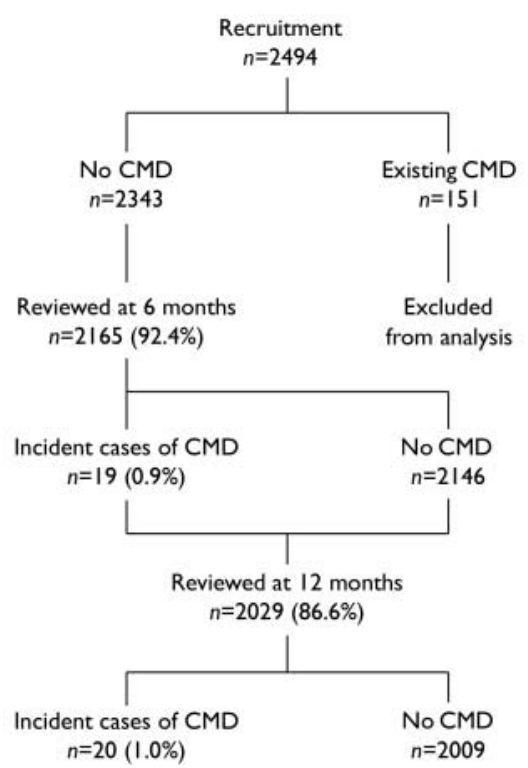

Fig. I Flow chart of participants in the study. CMD, common mental disorders.

risk factors and factors associated with spousal relationships were restricted to married participants. In the final model, which included all the women, variables with missing values had the values recoded as 9. For categorical variables with evidence of linearity, a $P$-value for trend is shown; otherwise the $P$-value shows the overall significance of the variable. Continuous variables such as age, household income, autonomy score, social integration score, family support score, physical disability, somatoform disorders score, CIS-R score, household size and income were converted to categorical variables (e.g. tertiles or quartiles) based on the distribution of scores. Reproductive tract infections were treated as a composite variable of any reproductive tract infection or any sexually transmitted infection (chlamydia, gonorrhoea, trichomoniasis). All significance tests are two-tailed.

\section{RESULTS}

Of the 3000 randomly selected women, 2494 consented to participate in the study $(83.1 \%)$. Details of the recruited sample have been published elsewhere (Patel et al, 2006). At the first review (mean review interval 24 weeks, s.d. 1.4 weeks), 2316 participants completed the review procedure $(92.9 \%)$. At the second review (mean review interval 52.2 weeks, s.d. 2.3 weeks) 2167 participants completed the review procedure $(86.9 \%)$. There was no difference between participants who were reviewed
Table I Association of baseline socio-economic characteristics with common mental disorders ( $n=2166$ unless otherwise stated)

\begin{tabular}{|c|c|c|c|}
\hline Baseline characteristics & $\begin{array}{c}\text { Incident cases of CMD' } \\
n(\%)\end{array}$ & $\begin{array}{c}\text { Univariate OR } \\
\qquad(95 \% \mathrm{Cl})\end{array}$ & $P$ \\
\hline \multicolumn{4}{|l|}{ Personal demographic data } \\
\hline \multicolumn{4}{|l|}{ Age (years) } \\
\hline $18-24$ & $4(0.9)$ & 1 & \\
\hline $25-29$ & $8(2.1)$ & $2.31(0.7-7.7)$ & 0.17 \\
\hline $30-34$ & $6(1.4)$ & I.5I (0.4-5.4) & 0.52 \\
\hline $34-40$ & II (2.6) & $2.93(0.9-9.3)$ & 0.07 \\
\hline $40-50$ & $10(2.0)$ & $2.26(0.7-7.2)$ & 0.17 \\
\hline \multicolumn{4}{|l|}{ Participant type } \\
\hline Randomised selection & $23(1.7)$ & 1 & \\
\hline Replacement & $16(1.9)$ & $1.09(0.6-2.1)$ & 0.78 \\
\hline \multicolumn{4}{|l|}{ Language } \\
\hline Konkani & $34(1.9)$ & $\mathrm{I}$ & \\
\hline Other languages & $5(1.5)$ & $0.80(0.3-2.1)$ & 0.65 \\
\hline \multicolumn{4}{|l|}{ Education (years) } \\
\hline None & $7(3.5)$ & I & \\
\hline $1-9$ & $21(2.5)$ & $0.71(0.3-1.7)$ & \\
\hline $10-14$ & $10(1.1)$ & $0.32(0.1-0.8)$ & \\
\hline $15-23$ & I (0.4) & $0.11(0.0-0.9)$ & $0.002^{2}$ \\
\hline \multicolumn{4}{|l|}{ Literate (read and write) } \\
\hline Yes & $31(1.6)$ & I & \\
\hline No & $8(2.8)$ & $1.72(0.8-3.8)$ & 0.17 \\
\hline \multicolumn{4}{|l|}{ Ethnicity } \\
\hline Goan & $34(1.7)$ & I & \\
\hline Migrant & $5(2.6)$ & I.5I (0.6-3.9) & 0.40 \\
\hline \multicolumn{4}{|l|}{ Religion } \\
\hline Hindu & $26(1.6)$ & $\mathrm{I}$ & \\
\hline Christian & $12(2.6)$ & $1.64(0.8-3.3)$ & 0.16 \\
\hline Muslim & I (I.5) & $0.94(0.1-7.0)$ & 0.95 \\
\hline \multicolumn{4}{|l|}{ Occupation } \\
\hline Homemaker & $33(2.3)$ & $\mathrm{I}$ & \\
\hline Employed & $4(0.9)$ & $0.38(0.1-I . I)$ & 0.07 \\
\hline Other & $2(0.8)$ & $0.33(0.1-1.4)$ & 0.13 \\
\hline \multicolumn{4}{|l|}{ Marital status } \\
\hline Single & $2(0.3)$ & 1 & \\
\hline Married & $35(2.3)$ & $6.67(1.6-27.8)$ & 0.009 \\
\hline Divorced/widowed/separated & $2(3.3)$ & 9.84 (I.4-7I.I) & 0.02 \\
\hline
\end{tabular}

Economic characteristics

Housing

Own home

Rented/other

Toilet access

In house

Outside house

Tap water in house

Yes

No
35 (1.8)

4 (I.9)

$1.04(0.4-2.9)$

0.94

12 (I.3)

27 (2.I)

$1.60(0.8-3.2)$

0.18

II (I.I)

0.52

78

6.65

$002^{2}$

16

07

0.02

28 (2.3) 
Table I (continued)

\begin{tabular}{|c|c|c|c|}
\hline Baseline characteristics & $\begin{array}{c}\text { Incident cases of CMD' } \\
n(\%)\end{array}$ & $\begin{array}{l}\text { Univariate OR } \\
\qquad(95 \% \mathrm{Cl})\end{array}$ & $P$ \\
\hline \multicolumn{4}{|l|}{ Per capita income, INR $(n=2165)$} \\
\hline$<2000$ & $23(3.2)$ & I & \\
\hline $2000-2999$ & $7(1.9)$ & $0.58(0.2-1.4)$ & \\
\hline $3000-4999$ & $5(0.9)$ & $0.28(0.1-0.7)$ & \\
\hline $5000+$ & $4(0.8)$ & $0.23(0.1-0.7)$ & $0.001^{2}$ \\
\hline \multicolumn{4}{|l|}{ Family in debt } \\
\hline No & $23(1.6)$ & I & \\
\hline Yes & $16(2.2)$ & $1.38(0.7-2.6)$ & 0.32 \\
\hline \multicolumn{4}{|l|}{ Hunger in the past 3 months } \\
\hline No & $34(1.6)$ & I & \\
\hline Yes & $5(5.3)$ & $3.37(1.3-8.8)$ & 0.01 \\
\hline \multicolumn{4}{|l|}{ Managing financially } \\
\hline No difficulty & $15(0.99)$ & I & \\
\hline Difficulty making ends meet & $24(3.7)$ & $3.81(2.0-7.3)$ & $<0.001$ \\
\hline
\end{tabular}

CMD, common mental disorder; INR, Indian rupee.

I. Women who developed a common mental disorder during the study (incident cases) indicate the risk for the particular baseline characteristic.

2. Trend.

and those who were lost to follow-up at either review on the following recruitment characteristics: education, household family income, CIS-R score, any reproductive tract infection or sexually transmitted infection, experiencing abnormal vaginal discharge, and self-reported chronic physical health problems. However, younger, unmarried participants were more likely to be lost to follow-up, because they had moved away from home for occupational, educational or marital reasons. Replacement participants had higher follow-up at both rounds (round 1: 94.2\% v. 92.1\%, $P=0.05$; round $2: 89.7 \%$ v. $85.2 \%$, $P=0.001$ ). Language was also associated; migrants who were non-Konkani speakers had lower follow-up at both rounds, mainly because they had left the community. Follow-up at round 2 was lower among illiterate participants.

At least one review was completed by 2317 women, 151 of whom had a common mental disorder at recruitment; the analyses presented are thus based on the sample of 2166 women who completed at least one review and did not have a common mental disorder at recruitment. The study flow chart is shown in Fig. 1. A total of 39 participants had an incident common mental disorder; 31 were diagnosed as having mixed anxiety-depressive disorder and 8 as having depressive disorder. Thus the overall 12-month rate of new episodes of common mental disorder was $1.8 \%$ (95\% CI $1.3-2.4 \%$ ).

\section{Socio-economic risk factors}

The baseline socio-economic factors most strongly associated with the risk for common mental disorders were related to deprivation and poverty, i.e. low level of education, low household income, lack of access to running water in the home, having experienced hunger and difficulties in making ends meet (Table 1). Compared with single women, married and divorced/ widowed women were at significantly elevated risk. When these baseline factors were combined in a multivariate model, the following remained significantly associated with common mental disorders: being married $(\mathrm{OR}=6.52$, 95\% CI 1.627.3); being divorced or widowed $(\mathrm{OR}=6.04,95 \%$ CI 0.8-44.3); higher total monthly household income $(\mathrm{OR}=0.37$, 95\% CI 0.1-1.1 for the highest quartile compared with the lowest); and the experience of difficulties in making ends meet $(\mathrm{OR}=2.82,95 \% \mathrm{CI}=1.4-5.6)$.

\section{Psychosocial risk factors}

Baseline factors indicative of gender disadvantage, i.e. younger age at marriage, concern about the husband's substance misuse habits, and violence from others (typically in-laws for married women and parents for single women), were found to be strongly associated with common mental disorders in univariate analyses, but not after adjustment for socio-economic factors. Three types of marital abuse were elicited; none was associated with common mental disorders in univariate analyses; a composite variable of any marital abuse was also not associated with common mental disorders $(\mathrm{OR}=1.82,95 \%$ CI $0.8-4.0)$. Baseline sub-case threshold psychological morbidity and symptoms of somatoform disorders at recruitment were associated with increased risk of common mental disorders, as were tobacco and alcohol use in the previous 3 months; these associations remained significant after adjustment for socio-economic factors (Table 2).

\section{Reproductive and physical health risk factors}

Having had a pregnancy, younger age at first pregnancy and having had multiple pregnancies were all associated with common mental disorders in univariate analyses, but were not significant after adjustment for socio-economic factors. Gynaecological complaints (vaginal discharge, lower abdominal pain, dysuria and irregular menstrual periods) were associated with an increased risk and these associations remained evident after adjustment for socio-economic factors; 294 participants reported a long-standing illness or disability and 287 of them described the nature of this illness. The most common illnesses were cardiovascular diseases (101), diabetes (25) and spinal or back disorders (25). These, and physical disability at baseline, were significantly associated with common mental disorders after adjustments. However, none of the baseline biological markers of poor physical health (anaemia, reproductive or sexually transmitted infections, hypertension or low or high BMI) was associated with increased risk, either in univariate analyses or after adjustment (Table 3).

\section{Final model}

In the final multivariate model (Table 4), the following baseline factors were significantly associated with the onset of common mental disorders: abnormal vaginal discharge, low household income, having difficulty making ends meet, being married, divorced or widowed, smoking cigarettes or chewing tobacco in the previous 3 months, suffering from a chronic illness and sub-threshold psychological morbidity. 
Table 2 Association of baseline gender disadvantage and mental health characteristics with common mental disorders $(n=2166)$

\begin{tabular}{|c|c|c|c|c|c|}
\hline $\begin{array}{l}\text { Baseline } \\
\text { characteristics }\end{array}$ & $\begin{array}{c}\text { Incident cases of } \\
\text { CMD', } n(\%)^{\prime}\end{array}$ & $\begin{array}{c}\text { Univariate OR } \\
(95 \% \mathrm{Cl})\end{array}$ & $P$ & Adjust $\mathrm{OR}^{2}$ & $P$ \\
\hline \multicolumn{6}{|c|}{ Age at marriage $(n=1532)$} \\
\hline$<18$ years & $6(4.96)$ & I & & I & \\
\hline $19-20$ years & $9(2.4)$ & $0.48(0.2-1.4)$ & & $0.52(0.2-1.5)$ & \\
\hline $21-24$ years & $14(2.5)$ & $0.49(0.2-1.3)$ & & $0.67(0.2-1.8)$ & \\
\hline $25+$ years & $6(1.2)$ & $0.24(0.1-0.8)$ & $0.03^{3}$ & $0.37(0.1-1.2)$ & $0.2^{3}$ \\
\hline \multicolumn{6}{|c|}{ Husband verbal abuse $(n=1533)$} \\
\hline No & $28(2.1)$ & 1 & & & \\
\hline Yes & $7(3.6)$ & $1.76(0.8-4.1)$ & 0.19 & & \\
\hline \multicolumn{6}{|c|}{ Husband physical abuse $(n=1533)$} \\
\hline No & $32(2.2)$ & I & & & \\
\hline Yes & $3(2.5)$ & I.II (0.3-3.7) & 0.86 & & \\
\hline \multicolumn{6}{|c|}{ Husband sexual abuse $(n=1533)$} \\
\hline No & $33(2.2)$ & I & & & \\
\hline Yes & $2(4.9)$ & $2.27(0.5-9.8)$ & 0.27 & & \\
\hline \multicolumn{6}{|c|}{ Concerned about husband's habits $(n=1533)$} \\
\hline No & $23(1.9)$ & I & & I & \\
\hline Yes & $12(4.1)$ & $2.24(1.1-4.6)$ & 0.03 & $1.69(0.8-3.5)$ & 0.15 \\
\hline \multicolumn{6}{|c|}{ Violence from others } \\
\hline No & $34(1.6)$ & 1 & & $\mathrm{I}$ & \\
\hline Yes & $5(4.8)$ & $3.04(1.2-7.9)$ & 0.02 & $2.23(0.8-5.9)$ & 0.11 \\
\hline \multicolumn{6}{|c|}{ Social integration } \\
\hline High & $16(2.3)$ & I & & & \\
\hline Medium & $10(1.4)$ & $0.60(0.3-1.3)$ & 0.20 & & \\
\hline Low & $13(1.8)$ & $0.76(0.4-1.6)$ & 0.48 & & \\
\hline \multicolumn{6}{|c|}{ Autonomy in decision-making } \\
\hline High & $10(1.3)$ & I & & & \\
\hline Medium & I7 (I.8) & $1.37(0.6-3.0)$ & 0.43 & & \\
\hline Low & $12(2.4)$ & $1.80(0.8-4.2)$ & 0.17 & & \\
\hline \multicolumn{6}{|c|}{ Support from family } \\
\hline Low & $9(2.4)$ & I & & & \\
\hline Medium & $14(2.3)$ & $0.97(0.4-2.3)$ & 0.94 & & \\
\hline High & $16(1.4)$ & $0.57(0.2-1.3)$ & 0.19 & & \\
\hline \multicolumn{6}{|c|}{ Somatoform symptom score } \\
\hline $0-1$ & $8(I . I)$ & I & & I & \\
\hline $2-3$ & $2(0.4)$ & $0.38(0.1-1.8)$ & 0.23 & $0.32(0.1-1.5)$ & 0.15 \\
\hline 4-7 & II (I.7) & $\mathrm{I} .47(0.6-3.7)$ & 0.41 & $1.07(0.4-2.7)$ & 0.88 \\
\hline $8-\max$ & $18(5.1)$ & $4.62(1.98-10.7)$ & $<0.001$ & $2.91(1.2-6.9)$ & 0.01 \\
\hline \multicolumn{6}{|c|}{ Psychological morbidity (CIS-R) scores } \\
\hline 0 & $17(1.2)$ & I & & I & \\
\hline $\mathrm{I}-2$ & $3(I . I)$ & $0.89(0.2-3.0)$ & & $0.77(0.2-2.7)$ & \\
\hline $3-4$ & $3(1.4)$ & $1.12(0.3-3.8)$ & & $1.00(0.3-3.5)$ & \\
\hline $5-8$ & $9(4.2)$ & $3.48(1.5-7.9)$ & & $2.74(1.2-6.3)$ & \\
\hline $8-11$ & $7(8.6)$ & $7.57(3.0-18.8)$ & $<0.001^{3}$ & $5.37(2.1-13.6)$ & $<0.001^{3}$ \\
\hline \multicolumn{6}{|c|}{ Tobacco use in last 3 months $(n=2094)$} \\
\hline No & $31(1.5)$ & I & & I & \\
\hline Yes & $4(9.3)$ & $6.68(2.2-19.8)$ & 0.001 & $3.79(1.2-11.6)$ & 0.02 \\
\hline \multicolumn{6}{|c|}{ Alcohol use in last 3 months $(n=2094)$} \\
\hline No & $33(1.6)$ & I & & I & \\
\hline Yes & $2(5.6)$ & $3.61(0.8-15.6)$ & 0.09 & $3.98(0.9-18.0)$ & 0.07 \\
\hline
\end{tabular}

CMD, common mental disorders; CIS-R, Revised Clinical Interview Schedule.

I. Women who developed a common mental disorder during the study (incident cases) indicate the risk for the particular baseline characteristic.

2. Adjusted for marital status, income, difficulty in making ends meet.

3. Trend.

\section{DISCUSSION}

We report findings of the first (to the best of our knowledge) population-based cohort study of risk factors for common mental disorders from a low-income country. Our study reports specifically on risk factors for common mental disorders in women aged between 18 and 50 years, the demographic group at the highest risk. Our main findings were that economic difficulties, being married or divorced or widowed, gynaecological morbidity (particularly experiencing abnormal vaginal discharge) and self-reported chronic illness were independent risk factors; however, none of the objective measures of physical health, notably those reflecting nutritional status and reproductive and sexually transmitted infections, were associated with common mental disorders. Substance misuse, in particular current tobacco use, and subthreshold psychological morbidity at baseline, were also associated with increased risk. We propose a conceptual framework (Fig. 2) to explain the pathways from risk factors to common mental disorders in women. In this framework, distal socioeconomic factors are independent risk factors and also exert some of their effect through more proximal factors, notably poor reproductive health, substance misuse and chronic illnesses.

\section{Social disadvantage and common mental disorders}

All previous findings concerning risk factors for common mental disorders in low- and middle-income countries have been based on cross-sectional surveys, from which it is difficult to interpret causal relationships. Our design of a cohort study in which we excluded participants with a common mental disorder at baseline from the risk factor analysis demonstrates that economic deprivation is an independent risk factor for common mental disorders in women in our population. The likely mechanisms through which this association is mediated are diverse (Patel \& Kleinman, 2003); for example, poorer women are more likely to suffer from adverse lifeevents, to live in crowded or stressful conditions, to have fewer occupational opportunities and to have chronic illnesses; all of these are recognised risk factors for common mental disorders. The fact that there is association between poverty and common mental disorders in countries which 
are extremely diverse in their economic strength suggests that relative poverty is arguably a key factor.

In view of the strong associations reported in cross-sectional surveys between domestic violence and common mental disorders (Kumar et al, 2005; Patel et al, 2006), we were surprised that this was not an independent risk factor after adjustment for poverty and marital status in our longitudinal analysis. Several reasons may explain this; the rates of exposure to domestic violence were lower in our sample than those reported for women in India (Jejeebhoy, 1998); and the association between domestic violence and poor mental health in surveys may be partly explained by recall bias, reverse causality or confounding by socio-economic deprivation. However, we did find a strong, and independent, association between being widowed, divorced or married and an increased risk for common mental disorder. We think that the most likely explanation for the association with being widowed or divorced is related to social isolation and stigma; and we suggest that the increased risk in married women is at least partly due to their having to cope with multiple roles and leading potentially more restricted lives in their marital homes.

\section{Gynaecological morbidity and common mental disorders}

Recent cross-sectional surveys have shown that psychological factors, including common mental disorders, are major risk factors (Prasad et al, 2003; Patel et al, 2005) for abnormal vaginal discharge, one of the most common health complaints in women in South Asia. Gynaecological complaints are often culturally determined somatic idioms of distress for women facing severe social disadvantage and psychological distress (Patel \& Oomman, 1999). Ethnographic studies in South Asia have reported that women typically attribute their gynaecological symptoms to tension in their lives and to symptoms of tiredness and weakness, which in turn are often associated with heavy physical work and social disadvantage (Bang \& Bang, 1994). Our study suggests that one mechanism for the association reported in cross-sectional analyses is that such symptoms, which may have a variety of aetiologies, lie along the pathway between long-term social and interpersonal difficulties and common mental disorder. The lack of association between
Table 3 Association of baseline reproductive and physical health characteristics with common mental disorders $(n=2166)$

\begin{tabular}{|c|c|c|c|c|c|}
\hline $\begin{array}{l}\text { Baseline } \\
\text { characteristics }\end{array}$ & $\begin{array}{l}\text { Incident cases of } \\
\mathrm{CMD}^{\prime}, n(\%)\end{array}$ & $\begin{array}{l}\text { Univariate OR } \\
\qquad(95 \% \mathrm{Cl})\end{array}$ & $P$ & Adjusted OR ${ }^{2}$ & $P$ \\
\hline \multicolumn{6}{|c|}{ Obstetric baseline characteristics } \\
\hline \multicolumn{6}{|c|}{ Ever pregnant } \\
\hline No & $4(0.6)$ & $\mathrm{I}$ & & $\mathrm{I}$ & \\
\hline Yes & $35(2.3)$ & $3.87(1.4-10.9)$ & 0.01 & $0.91(0.2-3.9)$ & 0.90 \\
\hline \multicolumn{6}{|c|}{ Age at first pregnancy ${ }^{3}(n=1507)$} \\
\hline$<20$ years & $9(4.3)$ & I & & I & \\
\hline $20-29$ years & $24(2.1)$ & $0.47(0.2-7.0)$ & 0.06 & $0.60(0.3-1.3)$ & 0.21 \\
\hline $30-39$ years & $2(1.5)$ & $0.34(0.1-1.6)$ & 0.17 & $0.48(0.1-2.3)$ & 0.36 \\
\hline \multicolumn{6}{|c|}{ Pregnancy past year ${ }^{3}$} \\
\hline No & $32(1.6)$ & I & & & \\
\hline Yes & $7(3.0)$ & $1.86(0.8-4.2)$ & 0.14 & & \\
\hline \multicolumn{6}{|c|}{ Number of pregnancies ${ }^{3}(n=1507)$} \\
\hline I & $10(1.5)$ & 1 & & 1 & \\
\hline 2 & $22(3.2)$ & $2.24(1.0-4.8)$ & 0.04 & $1.90(0.9-4.1)$ & 0.10 \\
\hline $3+$ & $3(2.0)$ & $1.37(0.4-5.0)$ & 0.64 & $1.14(0.3-4.2)$ & 0.89 \\
\hline \multicolumn{6}{|c|}{ Infertility in past year ${ }^{4}(n=1532)$} \\
\hline No & $31(2.2)$ & I & & & \\
\hline Yes & $4(3.0)$ & $1.39(0.5-4.0)$ & 0.54 & & \\
\hline \multicolumn{6}{|c|}{ Gynaecological symptoms } \\
\hline \multicolumn{6}{|c|}{ Vaginal discharge } \\
\hline No & $27(1.4)$ & I & & I & \\
\hline Yes & $12(4.2)$ & $3.03(1.5-6.0)$ & $<0.001$ & $3.49(1.7-7.1)$ & 0.001 \\
\hline \multicolumn{6}{|c|}{ Itching in genitals } \\
\hline No & $31(1.7)$ & 1 & & & \\
\hline Yes & $8(2.6)$ & $1.60(0.7-3.5)$ & 0.24 & & \\
\hline \multicolumn{6}{|l|}{ Pain in abdomen } \\
\hline No & $30(1.6)$ & I & & I & \\
\hline Yes & $9(3.2)$ & $2.06(0.96-4.4)$ & 0.06 & $1.92(0.9-4.1)$ & 0.09 \\
\hline \multicolumn{6}{|l|}{ Dysuria } \\
\hline No & $3 I(I .6)$ & I & & I & \\
\hline Yes & $8(4.2)$ & $2.77(1.2-6.1)$ & 0.01 & $2.32(1.0-5.2)$ & 0.04 \\
\hline \multicolumn{6}{|c|}{ Dyspareunia $(n=1533)$} \\
\hline No & $33(2.3)$ & I & & & \\
\hline Yes & $2(2.7)$ & $1.54(0.4-6.5)$ & 0.56 & & \\
\hline \multicolumn{6}{|c|}{ Irregular menses $^{5}(n=1967)$} \\
\hline No & $7(0.98)$ & I & & I & \\
\hline Yes & $27(2.1)$ & $2.22(0.96-5.1)$ & 0.06 & $2.15(0.9-5.0)$ & 0.08 \\
\hline \multicolumn{6}{|c|}{ Menstrual cramps ${ }^{5}(n=1967)$} \\
\hline No & $12(1.3)$ & I & & & \\
\hline Yes & $22(2.1)$ & $1.59(0.8-3.2)$ & 0.20 & & \\
\hline \multicolumn{6}{|c|}{ Physical health indicators } \\
\hline \multicolumn{6}{|c|}{ Haemoglobin, g/dl $(n=2160)$} \\
\hline$<8$ & I (4.0) & I & & & \\
\hline $8-10.9$ & $5(1.4)$ & $0.33(0.03-2.9)$ & 0.31 & & \\
\hline $\mathrm{II+}$ & $33(1.9)$ & $0.45(0.1-3.5)$ & 0.45 & & \\
\hline \multicolumn{6}{|c|}{ Any RTI $(n=2073)$} \\
\hline Negative & $3 I(2 . I)$ & I & & & \\
\hline Positive & $6(1.0)$ & $0.49(0.2-1.2)$ & 0.11 & & \\
\hline
\end{tabular}


Table 3 Association of baseline reproductive and physical health characteristics with common mental disorders $(n=2166)$

\begin{tabular}{lccccc}
\hline Baseline & Incident cases & Univariate OR & $P$ & Adjusted OR & $P$ \\
characteristics & of CMD', $n(\%)$ & $(95 \% \mathrm{Cl})$ & & \\
\hline
\end{tabular}

Any RTI ( $n=2073)$

\begin{tabular}{|c|c|c|c|c|c|}
\hline Negative & $31(2.1)$ & 1 & & & \\
\hline Positive & $6(1.0)$ & $0.49(0.2-1.2)$ & 0.11 & & \\
\hline \multicolumn{6}{|c|}{ Any STI $(n=2102)$} \\
\hline Negative & $35(1.7)$ & 1 & & & \\
\hline Positive & $2(2.3)$ & $1.33(0.3-5.6)$ & 0.70 & & \\
\hline \multicolumn{6}{|c|}{ Body mass index $(n=15 \mid 0)$} \\
\hline$<17$ & $3(1.7)$ & I & & & \\
\hline $17-19$ & $8(2.5)$ & $1.47(0.4-5.6)$ & 0.57 & & \\
\hline $20-24$ & $17(2.5)$ & $1.49(0.4-5.1)$ & 0.53 & & \\
\hline $25+$ & $5(1.4)$ & $0.84(0.2-3.6)$ & 0.82 & & \\
\hline \multicolumn{6}{|c|}{ Systolic blood pressure $(n=1556)$} \\
\hline$\leqslant 100$ & $8(3)$ & 1 & & & \\
\hline $101-120$ & $19(1.9)$ & $0.61(0.3-1.4)$ & 0.25 & & \\
\hline $121 / 140$ & $5(2.3)$ & $0.75(0.2-2.3)$ & 0.62 & & \\
\hline$|4|+$ & $\mathrm{I}(\mathrm{I} .7)$ & $0.55(0.1-4.5)$ & 0.58 & & \\
\hline \multicolumn{6}{|c|}{ Diastolic blood pressure $(n=1556)$} \\
\hline$<70$ & $2(2.4)$ & 1 & & & \\
\hline $70-79$ & $20(2.2)$ & $0.91(0.2-3.9)$ & 0.89 & & \\
\hline $80-89$ & $10(2.2)$ & $0.94(0.2-4.4)$ & 0.94 & & \\
\hline $90+$ & $I(I)$ & $0.40(0.0-4.5)$ & 0.46 & & \\
\hline \multicolumn{6}{|c|}{ Chronic physical illness ( $n=2157)$} \\
\hline No & $27(1.4)$ & 1 & & I & \\
\hline Yes & $10(4.2)$ & $3.10(1.5-6.5)$ & 0.003 & $2.96(1.4-6.3)$ & 0.005 \\
\hline \multicolumn{6}{|c|}{ Physical disability scores } \\
\hline Low & $23(1.2)$ & 1 & & 1 & \\
\hline Moderate & $9(3.8)$ & $3.08(1.4-6.7)$ & & $2.59(1.2-5.7)$ & \\
\hline High & $7(7.5)$ & $6.41(2.7-15.3)$ & $<0.001^{6}$ & $4.37(1.8-10.7)$ & $<0.001^{6}$ \\
\hline
\end{tabular}

CMD, common mental disorders; RTI, reproductive tract infection; STI, sexually transmitted infection.

I. Women who developed a common mental disorder during the study (incident cases) indicate the risk for the particular baseline characteristic.

2. Adjusted for marital status, income, difficulty in making ends meet.

3. Women who have had at least one pregnancy

4. Married women only.

5. Women who are premenopausal, have not had a hysterectomy and are not lactating.

6. Trend.

biological indicators of reproductive health and common mental disorders suggests that the social contexts of gynaecological symptoms, including their possible impact on marital relationships, are the most plausible proximal mechanisms of association.

\section{Other risk factors}

Our study replicated the association of three well-defined risk factors for common mental disorder: sub-threshold psychological morbidity, tobacco use and chronic illness. A recent review confirms the high levels of comorbidity of physical and mental health problems and that this association is bidirectional (Evans et al, 2005).
Several mechanisms may explain this association, including common biological pathways for some chronic diseases and common mental disorder, the adverse effects of treatments for chronic diseases and the impact of pain and disability associated with chronic diseases on mental health. The latter is the most likely pathway; the association between physical disability and common mental disorders was markedly attenuated after adjustment for chronic physical illness. Substance use, of both alcohol and tobacco, was associated with common mental disorder, and the association with tobacco use (smoked or chewed) remained significant after adjustment for socio-economic and other risk factors. This finding replicates similar reports from longitudinal studies in developed countries which have reported these associations (Wagena et al, 2005). Several mechanisms might be considered to explain this association, but the fact that the association has been reported for the first time in a non-Western setting where tobacco use among women is relatively rare $(1.7 \%)$ and where most tobacco is chewed, points to the role of biological factors related to neuropharmacological effects of nicotine on neurotransmitter systems linked to depression (Breslau et al, 1998). Other plausible mechanisms can also be considered, for example confounding by unmeasured life difficulties which predict both tobacco use and common mental disorder. Unsurprisingly, in our study current psychological symptoms were associated with the risk of common mental disorders, which may be partly an artefact resulting from the dichotomisation into case and non-case categories of scores measuring the symptoms of depression and anxiety, which are typically continuously distributed in populations.

\section{Limitations and implications}

We did not measure biological indicators for chronic illnesses. However, we were able to measure indicators for locally relevant exposures reflecting nutrition and reproductive health. The overall participation rate in the study was high and the attrition rate was relatively low, enhancing our confidence in the generalisability of the findings. However, there might have been a selection bias at recruitment such that women with physical health problems were more likely to participate. In using a categorical approach based on a diagnostic algorithm to define our outcome, we will have missed an unknown number of women with clinically significant symptoms of common mental disorder which did not meet ICD-10 case criteria, i.e. sub-threshold morbidity which may be associated with adverse impact and helpseeking (Demyttenaere et al, 2004).

The implications of our findings are that public health and clinical interventions aimed at reducing the burden of common mental disorders in women must target those who are poor and facing acute economic problems. It is plausible that community development activities which enhance women's education and attenuate 
Table 4 Final multivariate model of association of socio-economic, reproductive and physical health risk factors with common mental disorders in women $(n=2166)$

\begin{tabular}{|c|c|c|}
\hline Baseline factor & Odds ratio & $P$ \\
\hline Abnormal vaginal discharge & $3.00(1.4-6.4)$ & 0.004 \\
\hline Chronic physical illness & $2.30(1.0-5.1)$ & 0.04 \\
\hline \multicolumn{3}{|l|}{ CIS-R score } \\
\hline 0 & 1 & \\
\hline $\mathrm{I}-2$ & $0.51(0.1-1.9)$ & \\
\hline $3-4$ & $0.71(0.2-2.5)$ & \\
\hline $5-8$ & $\mathrm{I} .8 \mathrm{I}(0.7-4.3)$ & \\
\hline $8-11$ & $3.10(1.1-8.5)$ & $0.03^{\prime}$ \\
\hline Tobacco use in past 3 months & $3.23(1.0-10.8)$ & 0.05 \\
\hline \multicolumn{3}{|l|}{ Marital status } \\
\hline Single & I & \\
\hline Married & $6.02(1.4-25.6)$ & 0.01 \\
\hline Widowed/separated/divorced & $6.23(0.8-47.8)$ & 0.08 \\
\hline Difficulties making ends meet & $2.39(1.2-4.9)$ & 0.02 \\
\hline \multicolumn{3}{|l|}{ Household per capita income, INR } \\
\hline$<2000$ & 1 & \\
\hline $2000-2999$ & $0.58(0.2-1.4)$ & \\
\hline $3000-4999$ & $0.36(0.1-I .0)$ & \\
\hline $5000+$ & $0.4 I(0.1-1.3)$ & $0.04^{\prime}$ \\
\hline
\end{tabular}

CIS-R, Revised Clinical Interview Schedule; INR, Indian rupee. I. Trend.

the impact of poverty will promote mental health. Advocacy is needed by global and national health-policy makers to highlight the greater vulnerability of the poor to common mental disorder and strengthen the capacity of health services to address these disorders. Screening may help identify women with common mental disorders, particularly in clinical settings such as gynaecological or medical clinics, where women with gynaecological symptoms and chronic illnesses seek help. However, such screening programmes must be twinned with effective management strategies for common mental disorders. Reproductive and primary healthcare must incorporate a strong emphasis on the assessment of the mental health and social circumstances of women with gynaecological symptoms or chronic illnesses. The threshold for interventions for common mental disorders may need revision; women with sub-threshold symptoms, at the very least, need closer follow-up to improve early detection and management. Women who are using tobacco are also a high-risk

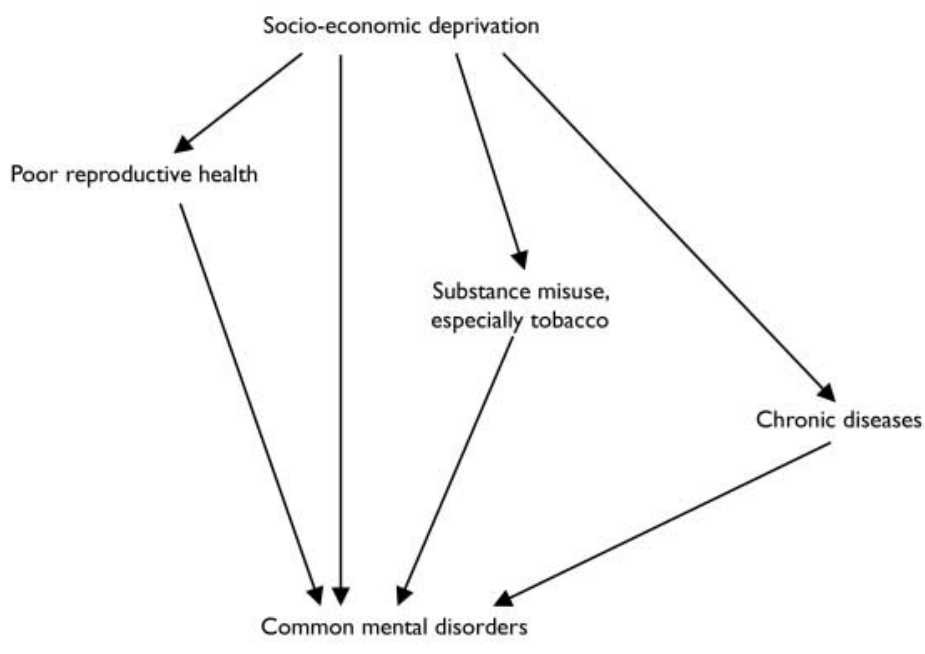

Fig. 2 Conceptual framework for pathways from risk factors to common mental disorders. group; interventions for early detection and treatment of common mental disorders should target women tobacco users. Further research is needed to identify the mechanisms through which some of the associations we have found are mediated; for example, what is the mechanism for the association between tobacco use and common mental disorders? Similarly, longitudinal studies examining the access and barriers to effective and affordable care for common mental disorders among poorer communities in low- and middleincome countries are needed; coping strategies which promote recovery and resilience may help identify mechanisms which promote mental health even in the context of economic adversity.

\section{ACKNOWLEDGEMENTS}

We are grateful to the Directorate of Health Services, Government of Goa, India, to the research team of the Stree Arogya Shodh Project which implemented this study in India and to the women who so willingly participated in this research.

This study was funded by a Wellcome Trust Career Development Fellowship in Clinical Tropical Medicine, to V.P.

\section{REFERENCES}

Bang, R. \& Bang, A. (1994) Women's perceptions of white vaginal discharge: ethnographic data from rural Maharashtra. In Listening to Women Talk about their Health: Issues \& Evidence from India (eds J. Gittelsohn, M. E. Bentley, P. J. Pelton, et al), pp. 79-94. New Delhi: Ford Foundation.

Breslau, N., Peterson, E. L., Schultz, L. R., et al (1998) Major depression and stages of smoking. A longitudinal investigation. Archives of General Psychiatry, 55, $161-166$.

Breslau, N., Schultz, L. R., Johnson, E. O., et al (2005) Smoking and the risk of suicidal behavior: a prospective study of a community sample. Archives of General Psychiatry, 62, 328-334.

Chaturvedi, S. \& Sarmukaddam, S. (1987)

Somatizers in psychiatric care. Indian Journal of Psychiatry, 29, 337-342.

Chwastiak, L. A. \& Von Korff, M. (2003) Disability in depression and back pain: evaluation of the World Health Organization Disability Assessment Schedule (WHO DAS II) in a primary care setting. Journal of Clinical Epidemiology, 56, 507-514.

Demyttenaere, K., Bruffaerts, R., Posada-Villa, J., et al (2004) Prevalence, severity, and unmet need for treatment of mental disorders in the World Health Organization World Mental Health Surveys, JAMA, 29I, $2581-2590$.

Evans, D. L., Charney, D. S., Lewis L., et al (2005) Mood disorders in the medically ill: scientific review and recommendations. Biological Psychiatry, 58, 175-189.

Goldberg, D. \& Huxley, P. (1992) Common Mental Disorders: A Biosocial Model. London: Tavistock/ Routledge. 
International Institute for Population Sciences (200I) National Family Health Survey-2, 1998-99: India. Mumbai: IIPS.

Jejeebhoy, S. (1998) Wife-beating in rural India: a husband's right? Evidence from survey data. Economic and Political Weekly, II April, 855-862.

\section{Jejeebhoy, S., Koenig, M. \& Elias, C. (2003)}

Reproductive Tract Infections and Other Gynaecological Disorders: A Multi-disciplinary Research Approach.

Cambridge: Cambridge University Press.

Krenzischeck, D. \& Tanseco, F. (1996) Comparative study of bedside and laboratory measurements of haemoglobin. American Journal of Critical Care, 5, $427-$

Kumar, S., Jeyaseelan, L., Suresh, S., et al (2005) Domestic violence and its mental health correlates in Indian women. British Journal of Psychiatry, 187, 62-67.

Lewis, G., Pelosi, A., Araya, R., et al (1992) Measuring psychiatric disorders in the community: a standardized assessment for use by lay interviewers. Psychological Medicine, 22, 465-486.

Meehan, M., Wawer, M. J., Serwadda, D., et al (2003) Laboratory methods for the diagnosis of reproductive tract infections and selected conditions in populationbased studies. In Reproductive Tract Infections and Other Gynaecological Disorders: A Multidisciplinary Approach (eds S. Jejeebhoy, M. Koenig \& C. Elias), pp. 26I-282. Cambridge: Cambridge University Press.

Patel, V., Pereira, J. \& Mann, A. (1998) Somatic and psychological models of common mental disorders in India. Psychological Medicine, 28, 135-143.

VIKRAM PATEL, MD, PhD, London School of Hygiene and Tropical Medicine, UK, and Sangath, Porvorim, Goa, India; BETTY R. KIRKWOOD, MA, MSc, FMedSci, London School of Hygiene and Tropical Medicine, UK; SULOCHANA PEDNEKAR, MA, Sangath, Porvorim, Goa, India; HELEN WEISS, PhD, DAVID MABEY, DM, FRCP, London School of Hygiene and Tropical Medicine, UK

Correspondence: Dr Vikram Patel, Sangath, 83I/I Alto-Porvorim, Goa, India 40352I. Fax: +91 832 24II709; email:Vikram.patel@Ishtm.ac.uk

(First received 28 January 2006, final revision 21 June 2006, accepted I August 2006)

Patel, V. \& Oomman, N. M. (1999) Mental health matters too: gynecological morbidity and depression in South Asia. Reproductive Health Matters, 7, 30-38.

Patel, V. \& Kleinman, A. (2003) Poverty and common mental disorders in developing countries. Bulletin of the World Health Organization, 81, 609-615.

Patel, V., Pednekar, S., Weiss, H., et al (2005) Why do women complain of vaginal discharge? A population survey of infectious and psychosocial risk factors in a South Asian community. International Journal of Epidemiology, 34, 853-862.

Patel, V., Kirkwood, B. R., Pednekar, S., et al (2006) Gender disadvantage and reproductive health risk factors for common mental disorder in women: a community survey in India. Archives of General Psychiatry, 63, 404-4I3.
Piccinelli, M. \& Wilkinson, G. (2000) Gender differences in depression. Critical review. British journal of Psychiatry, 177, 486-492.

Prasad, J., Abraham, S., Akila, B., et al (2003)

Symptoms related to the reproductive tract and menta health among women in rural Southern India. National Medical Journal of India, 16, 303-308.

Wagena, E. J., van Amelsvoort, L. G., Kant, I., et al (2005) Chronic bronchitis, cigarette smoking, and the subsequent onset of depression and anxiety: results from a prospective population-based cohort study. Psychosomatic Medicine, 67, 656-660.

World Health Organization (1992) International Statistical Classification of Diseases and Related Health Problems, Tenth Revision (ICD-10). Geneva: WHO.

World Health Organization (200I) The World Health Report 200I. Mental Health: New Understanding, New Hope. Geneva: WHO 